\title{
Rapid and sensitive trace gas detection with continuous wave Optical Parametric Oscillator-based Wavelength Modulation Spectroscopy
}

\author{
D.D. Arslanov • M. Spunei • A.K.Y. Ngai • \\ S.M. Cristescu • I.D. Lindsay · S.T. Persijn · K.J. Boller • \\ F.J.M. Harren
}

Received: 26 July 2010 / Revised version: 26 August 2010 / Published online: 25 September 2010

(C) The Author(s) 2010. This article is published with open access at Springerlink.com

\begin{abstract}
A fiber-amplified Distributed Bragg Reflector diode laser is used to pump a continuous wave, singly resonant Optical Parametric Oscillator (OPO). The output radiation covers the 3-4 $\mu \mathrm{m}$ with ability of rapid $(100 \mathrm{THz} / \mathrm{s})$ and broad mode-hop-free tuning $\left(5 \mathrm{~cm}^{-1}\right)$. Wavelength Modulation Spectroscopy is combined with the OPO to take optimal advantage of the spectral scan speed. The sensitivity of the system was determined as 0.8 ppbv (parts-perbillion by volume) for ethane $\left(\mathrm{C}_{2} \mathrm{H}_{6}\right)$ for the absorption peak at $2996.9 \mathrm{~cm}^{-1}$ recorded in 1.3 seconds, corresponding to a noise equivalent absorption sensitivity (NEAS) of $1.2 \times 10^{-9} \mathrm{~cm}^{-1} / \mathrm{Hz}^{1 / 2}$. A comparison between results using the $1^{\text {st }}, 2^{\text {nd }}$ and $4^{\text {th }}$ harmonic derivative signal from wavelength modulation was performed. The broad continuous tunability was demonstrated by covering $35 \mathrm{~cm}^{-1}$ while recording absorption features of ethane, methane and water.
\end{abstract}

D.D. Arslanov $(\bowtie) \cdot$ M. Spunei · A.K.Y. Ngai · S.M. Cristescu • F.J.M. Harren

Life Science Trace Gas Facility, Molecular and Laser Physics, Institute for Molecules and Materials, Radboud University, P.O. Box 9010, 6500 GL Nijmegen, The Netherlands e-mail: d.arslanov@ science.ru.nl

Fax: +31-24-3653311

M. Spunei · S.T. Persijn

VSL, Thijsseweg 11, 2629 JA Delft, The Netherlands

I.D. Lindsay

EPSRC Advanced Research Fellow Nanophysics and Soft Matter Group, H.H. Wills Physics Laboratory, University of Bristol, Tyndall Avenue, Bristol, BS8 1TL, UK

\section{K.J. Boller}

Laser Physics and Nonlinear Optics Group, Faculty of Science and Technology, University of Twente, P.O. Box 217, 7500 AE Enschede, The Netherlands

\section{Introduction}

To unravel highly dynamic processes in the life sciences there is an increasing need for time-resolved measurements of trace gas emissions. Examples of such processes include the release of aldehydes, alcohols, and acetates by plants subject to attack by herbivores [1, 2]; $\mathrm{CO}_{2}, \mathrm{H}_{2} \mathrm{O}$ and $\mathrm{CH}_{4}$ release in insect respiration [3]; or in humans, the kinetics of the gas composition of a single breath exhalation [4]. Capturing all details of the emission pattern requires an instrument which combines a high sensitivity at the ppbv level within a second time scale resolution or beyond. This goal has been partially achieved by several research groups using quantum cascade lasers in combination with spectroscopic techniques, such as cavity ring-down [5], frequency modulation, and wavelength modulation spectroscopy [6, 7]. However, most of these instruments only allow monitoring of a single gas due to the limited scanning range of the quantum cascade laser. Moreover, the time resolution is still an issue when second or sub-second time of measurement is required.

As many gases have strong absorptions in the midinfrared region, we chose to tackle this problem by using the well-developed tuning and modulation abilities of diode lasers in the near-infrared region and transfer these to the 3-4 $\mu \mathrm{m}$ range, by using a continuous wave SinglyResonant Optical Parametric Oscillator (SR-OPO) [7-11]. Such a combination is an ideal basis for trace gas detection by means of laser spectroscopy. Here, rapid and wide spectral tuning over $16.5 \mathrm{~cm}^{-1}$, rapid mode-hop-free tuning over $5 \mathrm{~cm}^{-1}$ (scan rate: $100 \mathrm{THz} / \mathrm{s}$ ), and broad continuous coverage over the complete $3-4 \mu \mathrm{m}$ range could be performed [9]. Additionally, these characteristics are combined with a high average power of $1 \mathrm{Watt}, 100 \mathrm{MHz}$ Optical Parametric Oscillator (OPO) linewidth, a long-term stability of 
$1.7 \times 10^{-3} \mathrm{~cm}^{-1}$ over 30 minutes and the ability to simply set and lock any wavelength within a pump tuning range. This SR-OPO could be combined with Direct Absorption Spectroscopy (DAS), which offers a simple mechanism to record trace gases. However it does not provide a high sensitivity [12] due to is short effective path length. Even by using of multiple pass cells optical path lengths cannot exceed a few hundreds of meters. To improve the sensitivity one can modulate the input signal with $\mathrm{kHz}-\mathrm{MHz}$ frequency and demodulate absorption signal by using a lock-in amplifier. With this method at least an order of magnitude is gained in sensitivity. In [7] Wavelength Modulation Spectroscopy (WMS) and Frequency Modulation Spectroscopy (FMS) techniques were demonstrated using a mid-IR OPO pumped by a fiber-amplified diode laser. In this paper, we extend this approach and present rapid and sensitive trace gas measurements by using a multi-pass cell in combination with WMS. This method provides rapid recordings at the ppbv level sensitivity for gases such as ethane, methane, hydrogen cyanide, etc. and opens up new applications in medical and life sciences [13].

\section{Experimental setup}

The experimental setup of the SR-OPO is shown in Fig. 1 and is described earlier [9], here only a short description is given. An $80 \mathrm{~mW}$ multi-section Distributed Bragg Reflector (DBR) diode laser (Eagleyard Photonics, linewidth $40 \mathrm{MHz}$ [9]) with an output wavelength of $1082 \mathrm{~nm}$ is used to seed a $30 \mathrm{~m}$ long double-clad $\mathrm{Yb}$-doped fiber amplifier pumped by a $25 \mathrm{~W}$ fiber-coupled $976 \mathrm{~nm} \mathrm{cw}$ diode laser bar. The output of the fiber amplifier $(7.5 \mathrm{~W})$ is used to pump the OPO. To suppress stimulated Brillouin scattering inside the fiber amplifier, a fast modulation ( $\geq 2 \mathrm{MHz}$ ) was applied to the gain section of the DBR laser, which resulted in a broadening of the output linewidth of this pump system to $100 \mathrm{MHz}[8,9]$.

The SR-OPO cavity $[9,11]$ consists of a four-mirror bowtie ring design with two concave mirrors $(R=10 \mathrm{~cm})$ and two flat mirrors, all being highly transparent for pump and idler, and highly reflective for the signal wavelength. The pump beam was focused using a $75 \mathrm{~mm} \mathrm{CaF}_{2}$ lens in a $5 \% \mathrm{MgO}$-doped PPLN crystal (HC Photonics) with a length of $5 \mathrm{~cm}$ and with seven poling periods ranging from 28.5 to $31.5 \mu \mathrm{m}$. To suppress etalon effects one of the two edges had a slight wedge of 1 degree and both sides were antireflective coated for the pump, signal and idler wavelengths. The crystal was placed in an oven capable of maintaining temperatures from 20 to $200^{\circ} \mathrm{C}$ with a stability of $0.02^{\circ} \mathrm{C}$. In some of the experiments an uncoated $400-\mu m$-thick solid YAG etalon (free spectral range $207 \mathrm{GHz}$ ) was inserted in the OPO cavity to enhance long-term frequency stability. Up to $1 \mathrm{~W}$ of idler output was produced at a pump power of $7.5 \mathrm{~W}$ with a power stability better than $3 \%$.

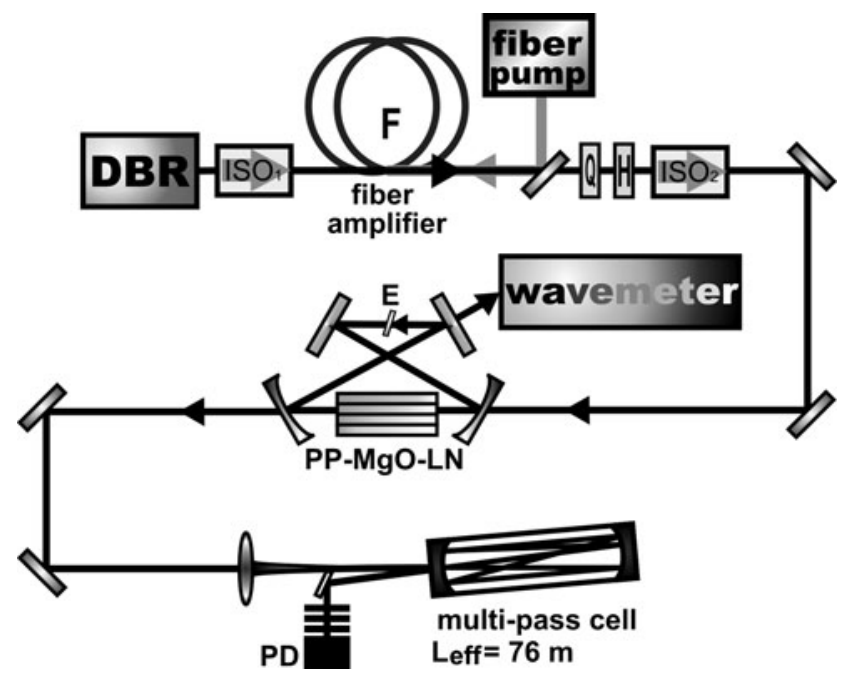

Fig. 1 Experimental setup. DBR Distributed Bragg Reflector diode laser, ISO 1 and 2 isolators (to reduce feedback into the DBR laser), $F$ double-clad Yb-doped fiber, $Q$ quarter-wave-plate, $H$ half-wave-plate, $P P-M g O-L N \mathrm{MgO}$-doped periodically poled lithium niobate crystal, $E$ intra-cavity etalon, multi-pass cell, $P D$ photodetector

Tuning of the OPO was performed in three steps. The first step was selecting an appropriate poling period of the PPLN crystal to roughly adjust the idler frequency at a wavelength between 3 and $4 \mu \mathrm{m}$. Then, by changing the crystal temperature the output wavelength can be changed within a $200 \mathrm{~cm}^{-1}$ range [10]; thirdly a fast, $5 \mathrm{~cm}^{-1}$-range, mode-hop-free tuning can be achieved with the diode DBR pump laser. The latter is achieved by synchronously sweeping the current through resistive heaters of the DBR and the phase sections of the multi-section diode laser. Modehop-free tuning or the absence of multimode operation of the idler is confirmed by the absence of discontinuities or broadened absorption lines, respectively, in the measured gas absorption spectra. By choosing the appropriate amplitudes and DC offsets the mode-hop-free range can be shifted within a total range of $16.5 \mathrm{~cm}^{-1}$ [7, 8]. For fast scans the relative idler frequency was monitored using a 5-cm-thick germanium $(\mathrm{Ge})$ etalon (see Fig. 2a) $(\mathrm{FSR}=$ $1.1 \mathrm{GHz}$ ). For 'slow' experiments a wavelength meter was used (Bristol Instruments 621A) with a repetition rate of $2 \mathrm{~Hz}$.

To benefit from the fast scanning capabilities, the OPO was combined with wavelength modulation spectroscopy in a multi-pass absorption cell [14]. Using an anti-reflective coated $(<0.5 \%) f=50 \mathrm{~cm} \mathrm{ZnSe} \mathrm{lens,} \mathrm{the} \mathrm{idler} \mathrm{beam} \mathrm{was}$ focused into the multi-pass cell (Aerodyne, AMAC-76) with an absorption path length of $76 \mathrm{~m}$ and a volume of $300 \mathrm{ml}$. The original entrance window of the cell was replaced with an anti-reflective coated window to suppress etalon effects. The gas flow mixture was continuously flushed through this absorption cell. To maintain a $\sim 100$ mbar pressure inside the cell a pressure controller and a needle valve are used, 


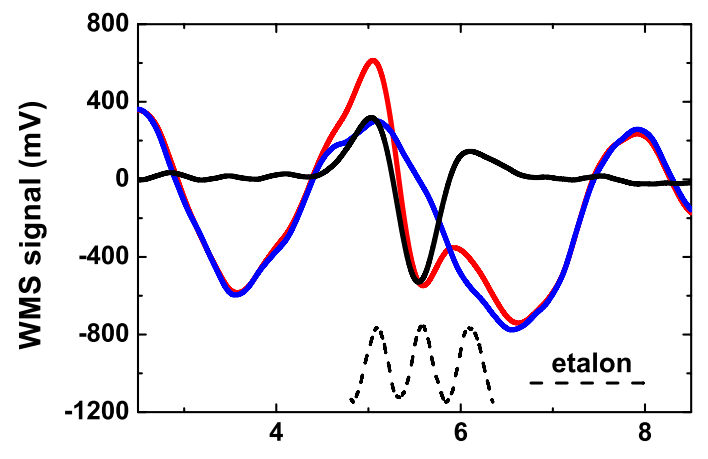

a

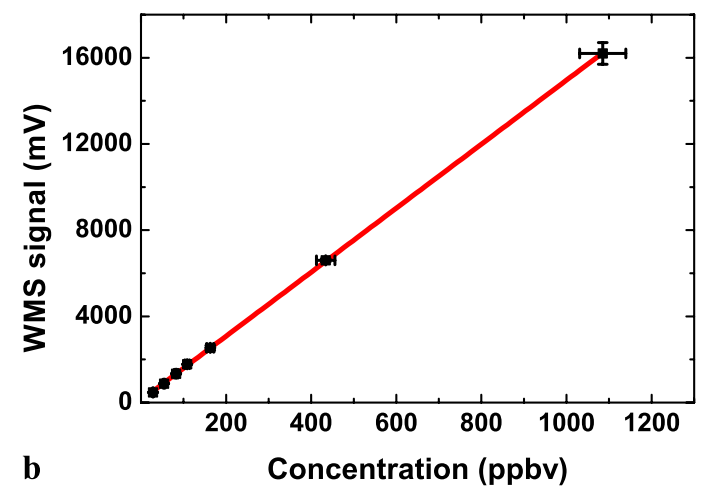

Fig. 2 (a) Example of the $2^{\text {nd }}$ harmonic wavelength modulation signal while scanning across a $50 \mathrm{ppbv}$ ethane absorption peak at $2996.9 \mathrm{~cm}^{-1}$. The red line represents the raw data, the blue line shows the background signal and the black solid line is the data with the background subtracted. The dashed line is the fringes pattern from the 5-cm-thick Ge etalon with FSR $\sim 1.1 \mathrm{GHz}$ and FWHM $\sim 500 \mathrm{MHz}$. (b) Second derivative WMS signal due to ethane peak at $2996.9 \mathrm{~cm}^{-1}$ measured at different concentrations between 28 and 1085 ppbv. A linear dependence on the concentration is shown with the fitted red line. Each concentration was measured three times. The detection limit determined from the data is $0.8 \mathrm{ppbv}$ over 1.3 second, corresponding to a NEAS of $1.2 \times 10^{-9} \mathrm{~cm}^{-1} / \mathrm{Hz}^{1 / 2}$

respectively in the entrance flow to the cell (Brooks Instrument 0154 and 5850) and in the exit flow towards the vacuum pump (Pfeiffer Vacuum MVP 015-4).

After the cell, the idler beam was detected with a fast $\mathrm{HgCdZnTe}$ thermo-electrically cooled photodetector ( $1 \mathrm{MHz}$ bandwidth, VIGO System PDI-2TE-4). To prevent saturation of the detector, the idler beam power of the OPO was reduced by using a pair of diaphragms. For the wavelength modulation experiments we used a $25 \mathrm{kHz}$ modulation frequency on top of the $2 \mathrm{MHz}$ applied to the DBR diode laser. The detected signal was demodulated with a lock-in amplifier (Stanford Research Systems SR830 DSP) at the first, second and fourth harmonic, and recorded with an oscilloscope (Tektronix TDS3032B) connected to the PC and data acquisition card (National Instruments PCI6229).

\section{Results and discussion}

Scans were made over an ethane $\left(\mathrm{C}_{2} \mathrm{H}_{6}\right)$ absorption feature at $2996.9 \mathrm{~cm}^{-1}$ to determine the sensitivity of the wavelength modulation system and to demonstrate the linear response at different concentrations. For this we used a calibrated mixture of $21.7 \mathrm{ppmv} \pm 5 \%$ (parts-per-million by volume) $\mathrm{C}_{2} \mathrm{H}_{6}$ in nitrogen (Air Liquide). To fill the multiple pass with calibrated concentrations down to the low partsper-billion level (ppbv) two mass flow controllers (Brooks Instrument, model $5850,5 \mathrm{l} / \mathrm{h}$ ) were used to dynamically dilute the calibrated ethane mixture with pure nitrogen (Air Liquide), varying the flow rates through the mass flow controllers. To even dilute the mixture further a second stage of two mass flow controllers was used at the exit of the first pair. In such a way the applied ethane concentration could be diluted in a controlled way by a factor $10^{3}$, meanwhile keeping a constant pressure inside the detection cell which was usually set in the range of 100-300 mbar during different experiments. With a cell volume of $300 \mathrm{ml}$ and an operating pressure of $250 \mathrm{mbar}$ this results in an effective gas volume of $75 \mathrm{ml}$. For a flow rate of $5 \mathrm{l} / \mathrm{h}$ this corresponds to a ventilation time of $54 \mathrm{~s}$.

Figure 2a shows the second derivative signal for the absorption line of $50 \mathrm{ppbv}$ ethane with and without background signal subtraction. For this, the phase and DBR sections of the diode laser were synchronously scanned by applying a $100 \mathrm{~Hz}$ saw tooth signal to each of the sections with the appropriate amplitude and DC offsets. Signals represent a 128 sweep average containing 10000 points collected on the oscilloscope in $1.3 \mathrm{~s}$. The strong interference fringes in the background signal are caused by overlap of the beam spots within the multi-pass cell. Even utilizing antireflection coated optical components such as windows and lenses, a time-dependent variation was observed in the background signal during frequency scans. The background was, therefore, measured with a pure nitrogen fill in the multipass cell before each new concentration point was recorded and then subsequently subtracted.

The ethane absorption was determined by the peak-peak value of the second derivative at different concentrations ranging from 28 to 1085 ppbv (Fig. 2b). WMS does not provide a real absorption signal; the harmonic component is directly proportional to the species concentration [15]. For each concentration, three measurement points were taken representing an average of 128 sweeps over the absorption feature. The linear fit with an $R$-square value of 0.9998 can be observed over a large dynamic range of concentrations. The detection limit $(1 \sigma)$ of the WMS recordings was determined as $0.8 \mathrm{ppbv}$ in 1.3 second for the 76 meter absorption path length, which corresponds to a NEAS of $1.2 \times 10^{-9} \mathrm{~cm}^{-1} / \mathrm{Hz}^{1 / 2}$ [16]. This proves that the OPO 


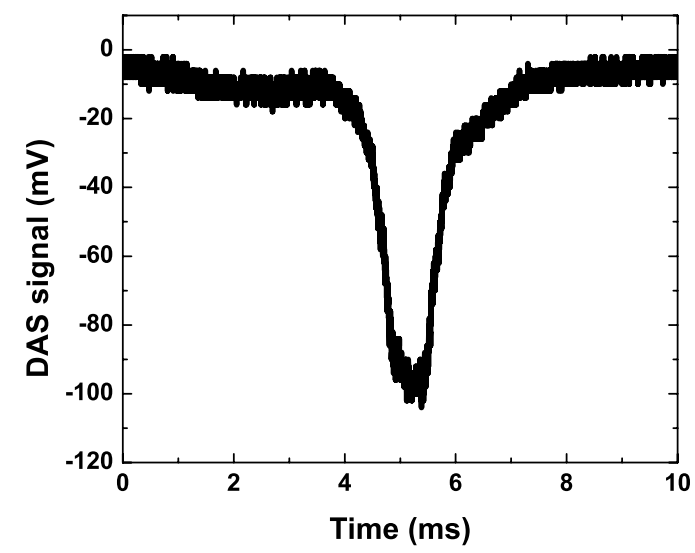

Fig. 3 DAS signal of 2 ppmv of ethane at $2996.9 \mathrm{~cm}^{-1}$ measured by using a 76-meter multiple pass cell. The signal is collected from 1000 averages recorded in $1 \mathrm{~s}$
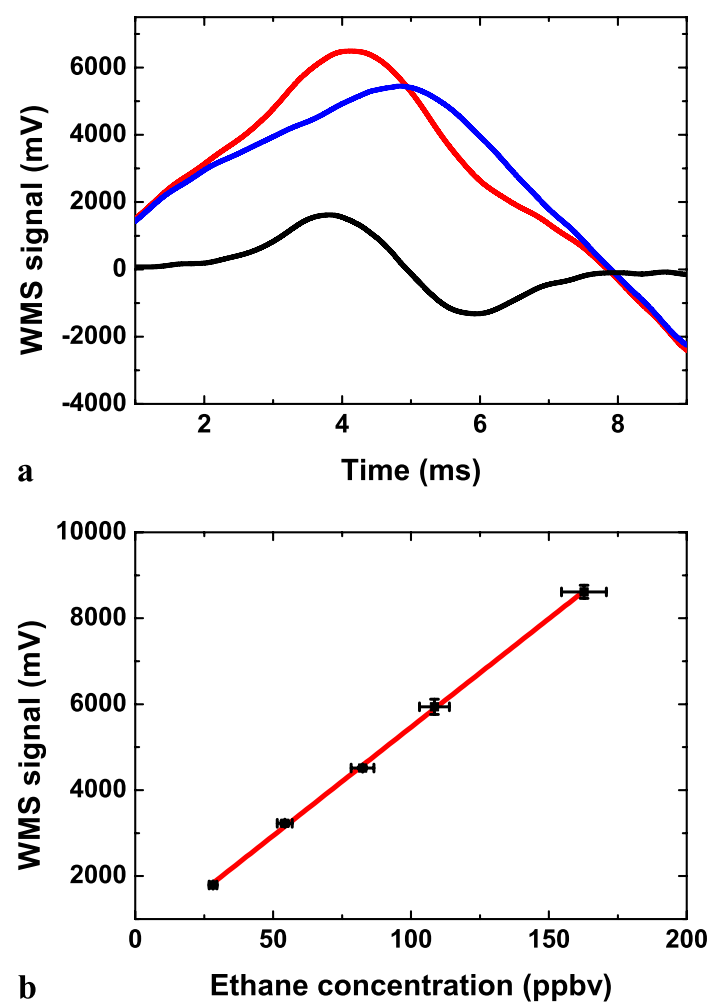

Fig. 4 (a) Example of the $1^{\text {st }}$ harmonic WMS signal while scanning across a 50 ppbv ethane absorption peak at $2996.9 \mathrm{~cm}^{-1}$. The red line represents the raw data, the blue line shows the background signal and the black line is the data with the background subtracted. (b) First derivative WMS signal due to the ethane peak at $2996.9 \mathrm{~cm}^{-1}$ measured at different concentrations between 28 and 160 ppbv. A linear dependence on the concentration is shown with the fitted red line. Each concentration was measured three times. The detection limit determined from the data is $0.9 \mathrm{ppbv}$ over 1.3 second, corresponding to a NEAS of $1.3 \times 10^{-9} \mathrm{~cm}^{-1} / \mathrm{Hz}^{1 / 2}$

based WMS is a powerful tool for a trace gas detection, providing simultaneously with a ppbv level of sensitivity and a second time resolution.
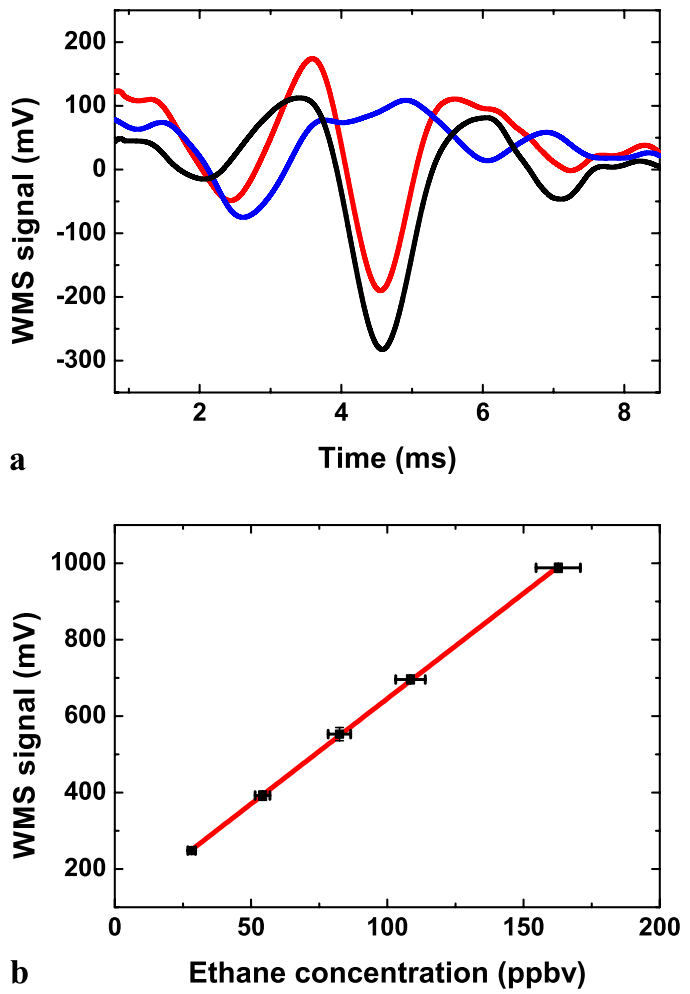

Fig. 5 (a) Example of the $4^{\text {th }}$ harmonic WMS signal while scanning across a 50 ppbv ethane absorption peak at $2996.9 \mathrm{~cm}^{-1}$. The red line represents the raw data, the blue line shows the background signal and the black line is the data with the background subtracted. (b) Fourth derivative WMS signal due to ethane peak at $2996.9 \mathrm{~cm}^{-1}$ measured at different concentrations between 28 and 160 ppbv. A linear dependence on the concentration is shown with the fitted red line. Each concentration was measured three times. The detection limit determined from the data is $0.9 \mathrm{ppbv}$ over 1.3 second, corresponding to a NEAS of $1.3 \times 10^{-9} \mathrm{~cm}^{-1} / \mathrm{Hz}^{1 / 2}$

Using only direct absorption spectroscopy (DAS) with the 76-meter multi-pass cell we achieved a detection limit of $\sim 100$ ppbv ( $\sim 1000$ scans, acquisition time $1 \mathrm{~s})$. As an example of DAS measurements 2 ppmv absorption signal of $\mathrm{C}_{2} \mathrm{H}_{6}$ is shown on the Fig. 3. This particular absorption feature consists of dozens of individual lines which are not resolved under the conditions used in the experiments here. This also has an effect on the WM measurements which do not show the nice pictures that you normally have when measuring individual lines. In spite of the 125 times worse sensitivity DAS has some advantages over WMS: the lack of a lock-in amplifier and the possibility of having of a laser scanning rate, limited only by the laser. In WMS, the laser scanning rate is limited, because the modulation frequency has to be orders of magnitude higher than the scanning frequency for best performance. In our experiments with WMS we have used a $100 \mathrm{~Hz}$ laser scanning rate instead of the potentially available few kHz. As such, for a high gas concentration and the necessity of a fast time response DAS could be advantageous. 
Fig. 6 (a) Wide-range WMS recorded using $2^{\text {nd }}$ harmonic detection. The 100 mbar gas in the multi-pass cell consisted of $5.9 \mathrm{ppmv}$ ethane, $1.2 \mathrm{ppmv}$ methane and $0.9 \%$ water. The $35.4 \mathrm{~cm}^{-1}$ range was covered by making three pump laser scans at different PP-MgO-LN crystal temperatures to shift the idler wavelength to another spectral window. (b) Calculated spectrum based on HITRAN data for 5.9 ppmv ethane (red line), 1.2 ppmv methane (black line) and $0.9 \%$ water (blue line)

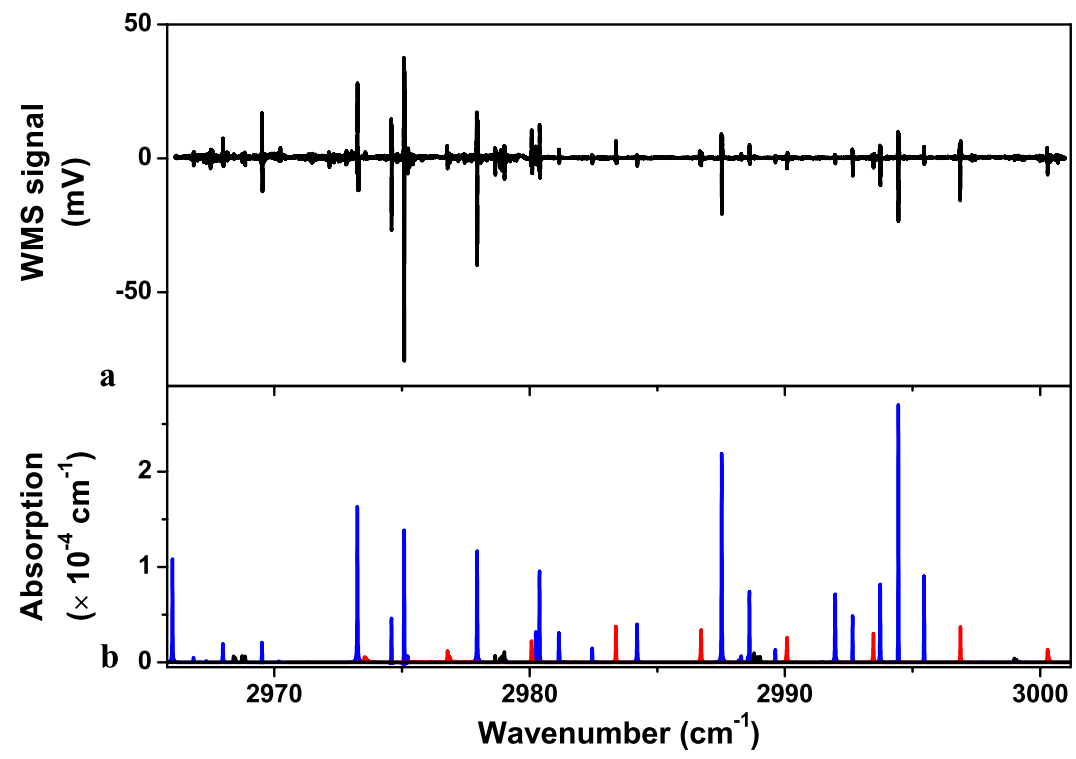

For comparison, we performed next to the $2^{\text {nd }}$ harmonic detection in WMS additional experiments using the $1^{\text {st }}$ and $4^{\text {th }}$ harmonic absorption derivatives. Figure $4 \mathrm{a}$ and Fig. 5a show examples of $50 \mathrm{ppbv}$ for the first and fourth derivatives signal of ethane at the same wavelength $\left(2996.9 \mathrm{~cm}^{-1}\right)$. Figure $4 \mathrm{~b}$ and Fig. $5 \mathrm{~b}$ show the linear response (with their linear fits) of the signal for the range of concentrations from 28 to $160 \mathrm{ppbv}$ for the first and fourth harmonics, respectively. These measurements were done at 160 mbar pressure inside the absorption cell and the scanning rate of the laser of $100 \mathrm{~Hz}$. This allows determining the detection limit as 0.9 ppbv of ethane in 1.3 second for both cases, giving comparable results with the second derivative experiment of a noise equivalent absorption sensitivity of $1.3 \times 10^{-9}$ $\mathrm{cm}^{-1} / \mathrm{Hz}^{1 / 2}$.

Although we used various parameters (time constant, pressures) in order to measure the $1^{\text {st }}, 2^{\text {nd }}$ and $4^{\text {th }}$ harmonics with the lock-in amplifier we get similar results in NEAS values, the signals are higher at the $1^{\text {st }}$ harmonic, the noise values increase proportionally, resulting in comparable signal-to-noise values. Besides this, depending on the background fringe pattern periodicity (caused by the etalon effect between the mirrors in the detection cell) it is-in our case - more advantageous to measure at the $2^{\text {nd }}$ and $4^{\text {th }}$ harmonic due to the higher contrast factor. This can be easily seen comparing Figs. 2a, 4a and 5a. A concentration of 50 ppbv ethane was measured in all cases, but the raw signal of the $4^{\text {th }}$ harmonic (red line Fig. 5a) intuitively gives more information about the real absorption signal than the raw $2^{\text {nd }}$ harmonic signal (red line Fig. 2a) and even more than the $1^{\text {st }}$ (red line Fig. 4a). It can be useful to work with the $4^{\text {th }}$ harmonic because of the presence of many time-dependent variables in the experiment, such as pressure and temperature of the cell, gas flow mixture into the cell, wavelength stability of the laser, etc. To be more precise and to be able to compare various results we have measured regularly the background signal between each concentration point to correct the data.

The above experiments are devoted to single gas detection, while in life sciences the study of physiological processes frequently requires monitoring of a number of gases, simultaneously. The advantage of using this OPO in comparison to other laser sources is its wide tunability from 3 to $4 \mu \mathrm{m}$, giving an opportunity to detect several gaseous compounds in one laser scan. The whole OPO tuning range can be used as the multi-pass cell is equipped with metallic mirrors with broadband reflection spectra from the visible to mid infrared. To extend the scanning range the crystal temperature is changed. To record a range of $35.4 \mathrm{~cm}^{-1}$ three pump laser scans (6.5 seconds per scan) were made at different PP-MgO-LN crystal temperatures. Figure 6a depicts the $2^{\text {nd }}$ harmonic signal of a 100 mbar mixture of lab air and ethane. In the spectrum absorption peaks are visible of ethane (5.9 ppmv), methane (1.2 ppmv) and water $(0.9 \%$, calibrated with a hygrometer). The spectra for these gases were also calculated based on HITRAN data [17] using a pseudo-Voigt profile [18], which are presented on the Fig. 6b.

\section{Conclusions}

The detection limit of $0.8 \mathrm{ppbv}$, measured in $1.3 \mathrm{sec}$ onds (NEAS of $1.2 \times 10^{-9} \mathrm{~cm}^{-1} / \mathrm{Hz}^{1 / 2}$ ) for ethane clearly demonstrates that a SR-OPO, pumped by a fiber-amplified diode laser, in combination with WMS using a multiple pass cell, can be a powerful tool for rapid and sensitive trace gas detection in the wavelength tuning range of the OPO between 3-4 $\mu \mathrm{m}$. Moreover, the approach allows recording 
over a wide spectral range (see Fig. 6), which offers attractive possibilities for capturing multi-component gas mixtures. Such utilities are necessary for applications within Life Science, which require a fast, sensitive detector with multi-component capabilities. The OPO enables recording of several individual absorption lines, spread over a wider spectroscopic range ignoring other spectroscopic features in between. In general the sensitivity of wavelength modulation spectroscopy is limited, due to the fringe patterns on the background signal that are induced by etalon effects between optic elements. This can be improved by recording the background signal and the total gas absorption signal, simultaneously. As such, most fringes and time-dependent fluctuations can be canceled out, thereby improving the sensitivity.

Acknowledgements This research was financially supported by the Dutch Technology Foundation STW and the European Commission under the program New and Emerging Science and Technologies, contract number FP6-NESTA-0025042 'Optical Nose'. I.D. Lindsay acknowledges support of the European Commission in the form of a Marie Curie Intra-European Fellowship.

Open Access This article is distributed under the terms of the Creative Commons Attribution Noncommercial License which permits any noncommercial use, distribution, and reproduction in any medium, provided the original author(s) and source are credited.

\section{References}

1. T.C.J. Turlings, U.B. Lengwiler, M.L. Bernasconi, D. Wechsler, Planta 207, 146 (1998)

2. A. Kessler, I.T. Baldwin, Science 291, 2141 (2001)
3. S.K. Hetz, T.J. Bradley, Nature 433, 516 (2005)

4. B.W.M. Moeskops, H. Naus, S.M. Cristescu, F.J.M. Harren, Appl. Phys. B 82, 649 (2006)

5. G. Berden, R. Peeters, G. Meijer, Int. Rev. Phys. Chem. 19, 565 (2000)

6. P. Werle, K. Maurer, R. Kormann, R. Mucke, F. D'Amato, T. Lancia, A. Popov, Spectrochim. Acta A 58, 2361 (2002)

7. I.D. Lindsay, P. Groß, C.J. Lee, A. Adhimoolam, K.J. Boller, Opt. Express 14, 12341 (2006)

8. A.K.Y. Ngai, S.T. Persijn, I.D. Lindsay, A.A. Kosterev, P. Gross, C.J. Lee, S.M. Cristescu, F.K. Tittel, K.J. Boller, F.J.M. Harren, Appl. Phys. B 89, 123 (2007)

9. I.D. Lindsay, B. Adhimoolam, P. Gross, M.E. Klein, K.J. Boller, Opt. Express 13, 1234 (2005)

10. A.K.Y. Ngai, S.T. Persijn, G. von Basum, F.J.M. Harren, Appl. Phys. B 85, 173 (2006)

11. M.M.J.W. van Herpen, S.E. Bisson, A.K.Y. Ngai, F.J.M. Harren, Appl. Phys. B 78, 281 (2004)

12. P. Werle, R. Mucke, F. Slemr, Appl. Phys. B 57, 131 (1993)

13. S.M. Cristescu, S.T. Persijn, S. te Lintel Hekkert, F.J.M. Harren, Appl. Phys. B 92, 343 (2008)

14. B.W.M. Moeskops, S.M. Cristescu, F.J.M. Harren, Opt. Lett. 31, 823 (2006)

15. P. Werle, Spectrochim. Acta A 54, 197 (1998)

16. E.J. Moyer, D.S. Sayres, G.S. Engel, J.M.St. Clair, F.N. Keutsch, N.T. Allen, J.H. Kroll, J.G. Anderson, Appl. Phys. B 92, 467 (2008)

17. L.S. Rothman, I.E. Gordon, A. Barbe, D. Chris Benner, P.F. Bernath, M. Birk, V. Boudon, L.R. Brown, A. Campargue, J.P. Champion, K. Chance, L.H. Coudert, V. Dana, V.M. Devi, S. Fally, J.-M. Flaud, R.R. Gamache, A. Goldman, D. Jacquemart, I. Kleiner, N. Lacome, W.J. Lafferty, J.-Y. Mandin, S.T. Massie, S.N. Mikhailenko, C.E. Miller, N. Moazzen-Ahmadi, O.V. Naumenko, A.V. Nikitin, J. Orphal, V.I. Perevalov, A. Perrin, A. Predoi-Cross, C.P. Rinsland, M. Rotger, M. Šimečková, M.A.H. Smith, K. Sung, S.A. Tashkun, J. Tennyson, R.A. Toth, A.C. Vandaele, J. Vander Auwera, J. Quantum Spectrosc. Radiat. Transf. 110, 533 (2009)

18. Y.Y. Liu, J.L. Lin, G.M. Huang, Y.Q. Guo, C.X. Duan, J. Opt. Soc. Am. B 18, 666 (2001) 